Pacific Journal of Mathematics

COMPLETE AREA MINIMIZING MINIMAL SURFACES JoEL HASS 


\title{
COMPLETE AREA MINIMIZING MINIMAL SURFACES WHICH ARE NOT TOTALLY GEODESIC
}

\author{
JoEL HaSS
}

\begin{abstract}
If a 3-manifold has non-negative Ricci curvature, then a complete area minimizing minimal surface in the 3-manifold is totally geodesic. The main theorem gives a method of constructing non-totally geodesic examples of such surfaces in certain manifolds which do not satisfy the Ricci curvature conditions. In particular, examples are described for hyperbolic space.
\end{abstract}

It has recently been shown by Fischer-Colbrie and Schoen, and independently by Do Carmo and Peng, that if $F$ is a complete stable minimal surface in $R^{3}$ then $F$ is a plane. Fischer-Colbrie and Schoen [FC-S] have obtained a similar result for 3-manifolds of non-negative Ricci curvature, showing that complete stable minimal surfaces in such manifolds are either totally geodesic planes or totally geodesic cylinders.

The corresponding result is false for general metrics on $R^{3}$. Anderson has obtained examples of complete stable minimal surfaces in hyperbolic 3-space, using the techniques of geometric measure theory [A]. However Anderson's methods do not specify the topological type of these examples. The main theorem of this paper will enable the construction of complete stable minimal surfaces which are not totally geodesic in a wide range of 3-manifolds. In fact the surfaces constructed will be area minimizing on any compact subset, a much stronger condition than stability. In hyperbolic space, we will obtain stable, embedded minimal surfaces whose limit sets are the entire sphere at infinity. The theorem is also applied to construct complete stable, embedded minimal surfaces which are not toally geodesic in another of the geometries on $R^{3}$, in which $R^{3}$ is metrically the product of hyperbolic 2 -space and the reals. Finally two examples are given of non-totally geodesic area minimizing minimal surfaces in non-simply connected 3-manifolds.

We will work in the category of smooth manifolds and maps. An immersed surface is minimal if the first variation of area is zero on any compact subset of $F$. A minimal surface $F$ is stable if the second variation of area is non-negative on any compact subset of $F$. A minimal surface $F$ is area minimizing if any compact subsurface $F_{1}$ of $F$ minimizes area in 
the class of all surfaces in $M$ homotopic to $F_{1}$ (rel $\left.\partial F\right)$. This in particular implies that the first non-zero derivative of area for any variation is positive, if it exists. A mapping of a compact surface $f: F \rightarrow M$ is called least area if it minimizes area in its homotopy class.

The first ingredient in the proof is a result of Freedman-Hass-Scott [F-H-S] saying that a compact cover of an area minimizing surface is also area minimizing.

LEMMA 1. Let $M$ be a $P^{2}$-irreducible 3-manifold which covers a compact Riemannian 3-manifold. Let $f: F \rightarrow M$ be a 2-sided map which induces an injection of the fundamental group. Let $M_{1}$ be a cover of $M$ and let $F_{1}$ be a finite $k$-fold cover of $F$ such that $f_{1}: F_{1} \rightarrow M_{1}$ is a lift of $f$. Then $f$ is least area if and only if $f_{1}$ is least area.

The next ingredient is a property of surfaces, called subgroup separability, which is proved for surface groups in [S].

LEMMA 2. Surface groups are subgroup separable.

This property is equivalent to a geometric condition, given in Lemma 1.4 , of [S], which we state below.

LEMMA 3. Let $Y$ be a Hausdorff topological space with a regular covering $\tilde{Y}$ and covering group $\Gamma$. Then $\Gamma$ is subgroup separable if and only if given a finitely generated subgroup $G$ of $\Gamma$ and a compact subset $X$ of $\tilde{Y} / G$ there is a finite cover $Y_{1}$ of $Y$ such that the projection $\tilde{Y} / G \rightarrow Y$ factors through $Y_{1}$ and $X$ projects injectively into $Y_{1}$.

THEOREM 1. Let $M$ be a $P^{2}$-irreducible Riemannian 3-manifold which covers a compact Riemannian 3-manifold and which admits a least area, 2-sided, incompressible surface $F$ that is not totally geodesic. Let $\tilde{M}$ be a cover of $M$ with the induced metric. Then $\tilde{M}$ contains an area minimizing complete not totally geodesic minimal surface. Furthermore, any lift of $F$ is such a surface.

Proof. Let $f: F \rightarrow M$ be a least area immersion which is not totally geodesic. Let $\tilde{F}$ be the cover of $F$ of smallest degree such that there exists a map $\tilde{f}: \tilde{F} \rightarrow \tilde{M}$ covering $f$. If $\tilde{F}$ is a finite cover of $F$, then $\tilde{f}$ is a least area immersion by Lemma 1 , and therefore is area minimizing. If $\tilde{F}$ is an infinite cover of $F$, suppose that it is not area minimizing. Then there is a compact subset $K$ of $\tilde{F}$ and a variation supported on $K$ such that the 
variation of area is negative. By Lemma 3 , there exists a space $F_{1}$ such that $\tilde{F}$ covers $F_{1}$ and $F_{1}$ is a finite cover of $F$, and such that the covering projection of $\tilde{F}$ to $F_{1}$ is 1-1 on $K$. Let $K_{1}$ be the projection of $K$ in $F_{1}$.

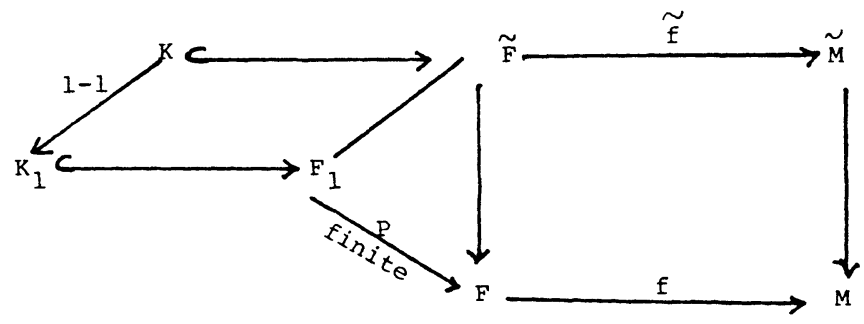

By Lemma $1, f_{1}=f \circ p$ is a least area immersion. But $K_{1}$ supports a variation yielding a mapping homotopic to $f_{1}$ but having less area. This contradiction shows that $\tilde{f}$ is indeed area minimizing.

REMARK. If we restrict to the case where $\tilde{M}$ is the universal cover of $M$ then the weaker condition of residual finiteness of surface groups would suffice.

REMARK. The condition of $P^{2}$-irreducibility is not necessary. If we take surfaces minimizing area in a class of surfaces having a given action on the fundamental group, rather than in a homotopy class, then a similar result holds.

We now look at some applications.

EXAMPLE 1. The first will take $\tilde{M}$ to be hyperbolic 3-space. For $M$ we take a compact hyperbolic 3-manifold which fibers over $S^{1}$. Examples of these were constructed by Jorgenson [J]. For $F$ we take a least area surface homotopic to a fiber. Such a surface exists and is embedded [F-H-S]. $\tilde{F}$ can be taken to be any lift of $F$. Then $\tilde{F}$ has the topological type of a plane, is embedded and is stable. Since $\pi_{1}(F)$ is a normal subgroup of $\pi_{1}(M)$ it follows that the two groups acting on hyperbolic 3-space as covering transformations have the same limit set on the sphere at infinity. But $M$ is compact and so $\pi_{1}(M)$ has dense limit set on the sphere at infinity. If $\tilde{F}$ were totally geodesic its limit set on the sphere at infinity would be a single circle. Cannon and Thurston have recently shown that the limit set of $\tilde{F}$ is a space filling curve.

EXAMPLE 2. For the next example consider $\tilde{M}=R^{3}$ with the metric obtained by taking the product of hyperbolic 2-space with the real line. Then $\tilde{M}$ covers $M$, a trivial circle bundle over a hyperbolic surface $S$ of 
genus greater than one, with the product metric. We can find in $M$ an embedded incompressible surface $F_{0}$, of genus greater than one, not homotopic to $S \times \mathrm{pt}$ or a cover of $S \times \mathrm{pt}$. As above, we can then minimize in the homotopy class of $F_{0}$ to obtain an embedded least area surface $F$. Since $F$ is not a torus or a cover of $S \times$ pt it must be tangent to $S \times p$ for some point $p$. If $F$, like $S \times p$, was totally geodesic, its image would then agree everywhere with that of $S \times p$, which would mean that $F$ covers $S \times p$. Thus $F$ satisfies the hypothesis of Theorem 1 .

EXAMPles 3 AND 4. For the final two examples we consider a pair of non-simply-connected spaces, each covering the $M$ of the previous example. We take $M_{1}$ to be the product of the hyperbolic plane with $S^{1}$ and $M_{2}$ to be the product of $S$ with the real line. In each case the arguments of the previous example apply to give area minimizing minimal surfaces which are not totally geodesic.

\section{REFERENCES}

[A] M. Anderson, Thesis, U. of California, Berkeley, 1981.

[C-T] J. Cannon and W. Thurston, to appear.

[D-P] M. DoCarmo and C. K. Peng, Stable complete minimal surfaces in $R^{3}$ are planes, Bull. Amer. Math. Soc., 1 (1979), 903-905.

[F-H-S] M. Freedman, J. Hass and G. P. Scott, Least area incompressible surfaces in 3-manifolds, to appear in Inventiones Math.

[J] T. Jorgenson, Compact 3-manifolds of constant negative curvature fibering over the circle, Ann. of Math., 106 (1977), 61-72.

[FC-S] D. Fischer-Colbrie and R. Schoen, The structure of complete stable minimal surfaces in 3-manifolds of non-negative scalar curvature, C.P.A.M., 33 (1980), 199-211.

[S] P. Scott, Subgoups of surface groups are almost geometric, J. London Math. Soc., (2), 17 (1978), 555-565.

Received October 5, 1982.

UNIVERSITY OF MICHIGAN

ANN ARbor, MI 48109 


\section{PACIFIC JOURNAL OF MATHEMATICS}

EDITORS

Donald BabBITT (Managing Editor)

University of California

Los Angeles, CA 90024

Hugo Rossi

University of Utah

Salt Lake City, UT 84112

C. C. Moore and Arthur Ogus

University of California

Berkeley, CA 94720
J. DugunduI

Department of Mathematics

University of Southern California

Los Angeles, CA 90089-1113

R. FINN and H. SAMELSON

Stanford University

Stanford, CA 94305

\section{ASSOCIATE EDITORS}
R. ARENS
E. F. BECKENBACH
B. H. NeumanN
F. WolF
K. YosHIDA (1906-1982)

\section{SUPPORTING INSTITUTIONS}

UNIVERSITY OF ARIZONA

UNIVERSITY OF BRITISH COLUMBIA

CALIFORNIA INSTITUTE OF TECHNOLOGY

UNIVERSITY OF CALIFORNIA

MONTANA STATE UNIVERSITY

UNIVERSITY OF NEVADA. RENO

NEW MEXICO STATE UNIVERSITY

OREGON STATE UNIVERSITY
UNIVERSITY OF OREGON

UNIVERSITY OF SOUTHERN CALIFORNIA

STANFORD UNIVERSITY

UNIVERSITY OF HAWAII

UNIVERSITY OF TOKYO

UNIVERSITY OF UTAH

WASHINGTON STATE UNIVERSITY

UNIVERSITY OF WASHINGTON 


\section{Pacific Journal of Mathematics}

\section{Vol. 111, No. $1 \quad$ November, 1984}

Harald Brandenburg and Adam Stefan Mysior, For every Hausdorff

space $Y$ there exists a nontrivial Moore space on which all continuous

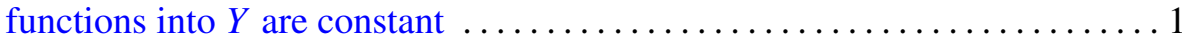

Henry Dappa, A Marcinkiewicz criterion for $L^{p}$-multipliers $\ldots \ldots \ldots \ldots \ldots 9$

P. H. Doyle, III and John Gilbert Hocking, Bijectively related spaces. I.

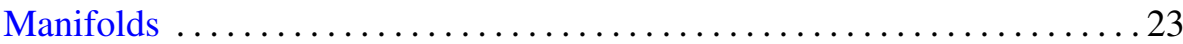

Joel Hass, Complete area minimizing minimal surfaces which are not totally

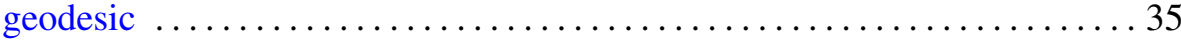

Aarno Hohti, On Ginsburg-Isbell derivatives and ranks of metric spaces .... 39

Richard Howard Hudson, Diophantine determinations of $3^{(p-1) / 8}$ and $5^{(p-1) / 4}$

A. F. Izé and A. Ventura, Asymptotic behavior of a perturbed neutral functional-differential equation related to the solution of the unperturbed

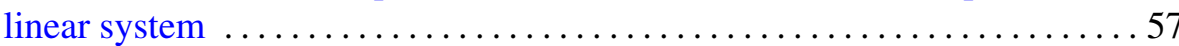

Palle E. T. Jorgensen, Spectral representations of unbounded nonlinear

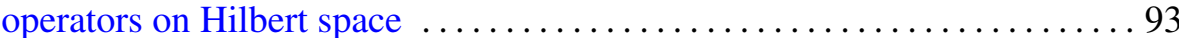

Darrell Conley Kent and Gary Douglas Richardson, Cauchy spaces with

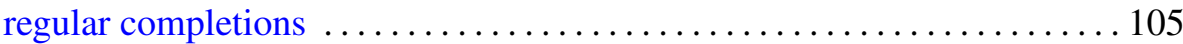

Mark Mahowald, An addendum to: "bo-resolutions" ................ 117

Stuart Wayne Margolis and Jean-Eric Pin, Minimal noncommutative varieties and power varieties

Carla Massaza and Alfio Ragusa, Some conditions on the homology groups of the Koszul complex

Vicente Miquel Molina, Some examples of Riemannian almost-product manifolds

Roderic Murufas, Inverse spectral problems for certain differential operators

Ulrich Oertel, Closed incompressible surfaces in complements of star links

Katsuro Sakai, A characterization of local equiconnectedness

William Victor Smith and Don Harrell Tucker, Weak integral convergence theorems and operator measures 